\title{
HYPERCOMMUTING VALUES IN ASSOCIATIVE RINGS WITH UNITY
}

\author{
VINCENZO DE FILIPPIS ${ }^{\bowtie}$ and GIOVANNI SCUDO \\ (Received 7 July 2011; accepted 17 October 2012; first published online 8 March 2013) \\ Communicated by B. Gardner
}

\begin{abstract}
Let $K$ be a commutative ring with unity, $R$ an associative $K$-algebra of characteristic different from 2 with unity element and no nonzero nil right ideal, and $f\left(x_{1}, \ldots, x_{n}\right)$ a multilinear polynomial over $K$. Assume that, for all $x \in R$ and for all $r_{1}, \ldots, r_{n} \in R$ there exist integers $m=m\left(x, r_{1}, \ldots, r_{n}\right) \geq 1$ and $k=k\left(x, r_{1}, \ldots, r_{n}\right) \geq 1$ such that $\left[x^{m}, f\left(r_{1}, \ldots, r_{n}\right)\right]_{k}=0$. We prove that: (1) if $\operatorname{char}(R)=0$ then $f\left(x_{1}, \ldots, x_{n}\right)$ is central-valued on $R$; and (2) if $\operatorname{char}(R)=p>2$ and $f\left(x_{1}, \ldots, x_{n}\right)$ is not a polynomial identity in $p \times p$ matrices of characteristic $p$, then $R$ satisfies $s_{n+2}\left(x_{1}, \ldots, x_{n+2}\right)$ and for any $r_{1}, \ldots, r_{n} \in R$ there exists $t=t\left(r_{1}, \ldots, r_{n}\right) \geq 1$ such that $f^{p^{t}}\left(r_{1}, \ldots, r_{n}\right) \in Z(R)$, the center of $R$.
\end{abstract}

2010 Mathematics subject classification: primary 16N60; secondary 16R20.

Keywords and phrases: prime and semiprime rings, associative algebras.

\section{Introduction}

Throughout this paper, $R$ always denotes an associative ring with unity and center $Z(R)$. The $k$ th commutator of $x, y \in R$, denoted by $[x, y]_{k}$ is defined inductively as follows: for $k=1,[x, y]_{1}=[x, y]=x y-y x$, and for $k>1,[x, y]_{k}=\left[[x, y]_{k-1}, y\right]$. In [1] Bergen proved that if $R$ is a ring with no nonzero nil right ideal and $f\left(x_{1}, \ldots, x_{n}\right)$ is a multilinear polynomial of degree $n$ which is not an identity for the $p \times p$ matrices in characteristic $p$, and for any $r_{1}, \ldots, r_{n} ; s_{1}, \ldots, s_{n} \in R$ there exist $m=m\left(r_{1}, \ldots, r_{n} ; s_{1}, \ldots, s_{n}\right) \geq 1$ and $t=t\left(r_{1}, \ldots, r_{n} ; s_{1}, \ldots, s_{n}\right) \geq 1$ such that $\left[f\left(r_{1}, \ldots, r_{n}\right)^{m}, f\left(s_{1}, \ldots, s_{n}\right)^{t}\right]=0$, then $R$ satisfies the standard identity $s_{n+2}\left(x_{1}, \ldots, x_{n+2}\right)$ and the values of $f\left(x_{1}, \ldots, x_{n}\right)$ are power central. In particular he showed that, if for any $r_{1}, r_{2}, s_{1}, s_{2} \in R$, there exist $m=m\left(r_{1}, r_{2}, s_{1}, s_{2}\right) \geq 1$ and $t=t\left(r_{1}, r_{2}, s_{1}, s_{2}\right) \geq 1$ such that $\left[\left[r_{1}, r_{2}\right]^{m},\left[s_{1}, s_{2}\right]^{t}\right]=0$, then $R$ satisfies the standard identity $s_{4}\left(x_{1}, \ldots, x_{4}\right)$.

Later, Chuang and Lin [5, Theorem 3] proved that if $R$ is a ring with no nonzero nil right ideals and for any $x, y \in R$ there exist $m=m(x, y) \geq 1$ and $t=t(x, y) \geq 1$ and $k=k(x, y) \geq 1$ such that $\left[x^{m}, y^{t}\right]_{k}=0$ then $R$ is commutative.

(c) 2013 Australian Mathematical Publishing Association Inc. 1446-7887/2013 \$16.00 
The aim of this note is to continue this line of investigation, combining in some sense the previous cited results and considering the $k$ th commutators involving the evaluations of a multilinear polynomial. Our main result will be the following theorem.

THEOREM 1.1. Let $K$ be a commutative ring with unity, $R$ an associative $K$-algebra of characteristic different from 2 with unity element and no nonzero nil right ideal, and $f\left(x_{1}, \ldots, x_{n}\right)$ a multilinear polynomial over $K$. Assume that, for all $x \in R$ and for all $r_{1}, \ldots, r_{n} \in R$ there exist integers $m=m\left(x, r_{1}, \ldots, r_{n}\right) \geq 1$ and $k=k\left(x, r_{1}, \ldots, r_{n}\right) \geq 1$ such that $\left[x^{m}, f\left(r_{1}, \ldots, r_{n}\right)\right]_{k}=0$. We prove the following results:

(1) if char $(R)=0$ then $f\left(x_{1}, \ldots, x_{n}\right)$ is central-valued on $R$;

(2) if $\operatorname{char}(R)=p>2$ and $f\left(x_{1}, \ldots, x_{n}\right)$ is not a polynomial identity in $p \times p$ matrices of characteristic $p$, then $R$ satisfies $s_{n+2}\left(x_{1}, \ldots, x_{n+2}\right)$ and for any $r_{1}, \ldots, r_{n} \in R$ there exists $t=t\left(r_{1}, \ldots, r_{n}\right) \geq 1$ such that $f^{p^{t}}\left(r_{1}, \ldots, r_{n}\right) \in Z(R)$, the center of $R$.

We would like to remark that in the case $\operatorname{char}(R)=p \neq 0$, the assumption that $f\left(x_{1}, \ldots, x_{n}\right)$ is not an identity in $p \times p$ matrices of characteristic $p$ is inherited from the fundamental work by Herstein et al. [8] where the structure of power central polynomials on division rings is determined under this hypothesis. We also note that a ring with no nonzero nil right ideal has no representation as a subdirect product of prime rings with the same property (unlike rings with no nonzero two-sided ideals). In order to circumvent this difficulty we will frequently make use of some methods contained in [1].

Firstly we fix some well-known facts.

FACT 1.2. Let $x, y \in R$. Then $[x, y]_{n}=\sum_{i=0}^{n}\left(\begin{array}{c}n \\ i\end{array}\right)(-1)^{i} y^{i} x y^{n-i}$ (here we put $\left.[x, y]_{0}=x\right)$.

FACT 1.3. Let $x, y, z \in R$. If $[x, y]_{n}=0$ for some $n \geq 1$ then $\left[x, y^{m}\right]_{n}=0$ for any $m \geq 1$ and $[x, y]_{q}=0$ for any $q \geq n$.

FACT 1.4. Let $x, y, z \in R$. If $\left[x, y^{m}\right]_{n}=0$ and $\left[z, y^{t}\right]_{n}=0$ then $\left[x, y^{m t}\right]_{n}=\left[z, y^{m t}\right]_{n}=0$.

We will also make use of the following results.

FАст 1.5. Let $R$ be a ring with no nonzero nil right ideal, and let $f\left(x_{1}, \ldots, x_{n}\right)$ be a multilinear polynomial in $n$ noncommuting variables. Assume that, for all $r_{1}, \ldots, r_{n} ; u_{1}, \ldots, u_{n} \in R$ there exist integers $m=m\left(r_{1}, \ldots, r_{n} ; u_{1} \ldots, u_{n}\right) \geq 1$ and $k=k\left(r_{1}, \ldots, r_{n} ; u_{1}, \ldots, u_{n}\right) \geq 1$ such that

$$
\left[f\left(r_{1}, \ldots, r_{n}\right)^{m}, f\left(u_{1}, \ldots, u_{n}\right)^{k}\right]=0 .
$$

If $\operatorname{char}(R)=p \neq 0$ and $f\left(x_{1}, \ldots, x_{n}\right)$ is not a polynomial identity in $p \times p$ matrices of characteristic $p$, then $R$ satisfies $s_{n+2}\left(x_{1}, \ldots, x_{n+2}\right)$ and for any $r_{1}, \ldots, r_{n} \in R$ there exists $t=t\left(r_{1}, \ldots, r_{n}\right) \geq 1$ such that $f^{p^{t}}\left(r_{1}, \ldots, r_{n}\right) \in Z(R)$ [1, Theorem 9].

FACT 1.6. Let $R$ be a $K$-algebra with no nonzero nil right ideal, and let $f\left(x_{1}, \ldots, x_{n}\right)$ be a multilinear polynomial over $K$. 
(1) If $f\left(x_{1}, \ldots, x_{n}\right)$ is nil in $R$, then $f\left(x_{1}, \ldots, x_{n}\right)$ is a polynomial identity for $R$ [3, Theorem 1].

(2) If $I$ is a right ideal of $R$ such that $f\left(x_{1}, \ldots, x_{n}\right)$ is nil in $I$, then $f\left(x_{1}, \ldots, x_{n}\right) x_{n+1}$ is a polynomial identity for $I$ (it is a consequence of [3, Main Theorem]).

FACT 1.7. Throughout this paper we denote

$$
\begin{aligned}
& H_{R}(f)=\left\{x \in R \mid \forall r_{1}, \ldots, r_{n} \in R \exists k=k\left(x, r_{1}, \ldots, r_{n}\right)\right. \\
& \text { such that } \left.\left[x, f\left(r_{1}, \ldots, r_{n}\right)\right]_{k}=0\right\},
\end{aligned}
$$

where $f\left(x_{1}, \ldots, x_{n}\right)$ is a multilinear polynomial in $n$ noncommuting variables. In particular, in the case where $R$ is a ring with no nonzero nil right ideal, and $\operatorname{char}(R)=0$, then the following hold.

(1) If $R$ is primitive and $R$ is not a division ring, then either $H_{R}(f)=Z(R)$ or $f\left(x_{1}, \ldots, x_{n}\right)$ is central valued in $R$ [6, Lemma 2.4].

(2) If $R$ is a domain such that $R=H_{R}(f)$, then $f\left(x_{1}, \ldots, x_{n}\right)$ is central valued in $R$ [6, Lemma 2.8].

\section{The results}

We begin with the following easy reduction.

Lemma 2.1. Let $\operatorname{char}(R)=p>2$. If $f\left(x_{1}, \ldots, x_{n}\right)$ is not a polynomial identity in $p \times p$ matrices of characteristic $p$, then $R$ satisfies $s_{n+2}\left(x_{1}, \ldots, x_{n+2}\right)$ and for any $r_{1}, \ldots, r_{n} \in R$ there exists $t=t\left(r_{1}, \ldots, r_{n}\right) \geq 1$ such that $f^{p^{t}}\left(r_{1}, \ldots, r_{n}\right) \in Z(R)$.

Proof. Given $x, r_{1}, \ldots, r_{n} \in R$, there exist suitable $m, k$ positive integers such that $\left[x^{m}, f\left(r_{1}, \ldots, r_{n}\right)\right]_{k}=0$. Hence, for $t \geq 1$ such that $p^{t} \geq k$,

$$
0=\left[x^{m}, f\left(r_{1}, \ldots, r_{n}\right)\right]_{p^{t}}=\left[x^{m}, f\left(r_{1}, \ldots, r_{n}\right)^{p^{t}}\right],
$$

and the conclusion follows from Fact 1.5.

In all that follows we will always assume that $\operatorname{char}(R)=0$, and moreover that $R$ has the unity element. Let $R_{\mathbb{Z}}$ be the localization of $R$ at $\mathbb{Z}$. By the multilinearity of $f\left(x_{1}, \ldots, x_{n}\right)$, our hypotheses on $R$ carry over to $R_{\mathbb{Z}}$. Therefore we may assume that $R$ is a $\mathbb{Q}$-algebra.

Lemma 2.2. Let $R$ be a domain. Then $f\left(x_{1}, \ldots, x_{n}\right)$ is central valued on $R$.

Proof. Pick $x \in R$ and $u=f\left(r_{1}, \ldots, r_{n}\right)$, with $r_{1}, \ldots, r_{n} \in R$. Notice that the set

$$
R_{u}=\left\{r \in R \mid \exists k=k(r, u) \geq 1 \text { such that }[r, u]_{k}=0\right\}
$$

is a subring of $R$. Moreover we observe that for any $x \in R$, there exists $m=m(x, u) \geq 1$ such that $x^{m} \in R_{u}$, that is $R$ is radical over $R_{u}$. By [2, Theorem 2], we have $R=R_{u}$. Therefore by the arbitrariness of $r_{1}, \ldots, r_{n} \in R$, it follows that for all $x \in R$ and for all $r_{1}, \ldots, r_{n} \in R$ there exists suitable $k \geq 1$ such that $\left[x, f\left(r_{1}, \ldots, r_{n}\right)\right]_{k}=0$. Hence by Fact 1.7 we have that $f\left(x_{1}, \ldots, x_{n}\right)$ is central valued on $R$. 
Lemma 2.3. If $R$ is primitive, then $f\left(x_{1}, \ldots, x_{n}\right)$ is central valued on $R$.

Proof. If $R$ is a division ring, then we conclude by Lemma 2.2. However we know that $R$ is a ring dense of $D$-linear transformations over $V$, where $D$ is a division ring and $V$ is a faithful irreducible right $R$-module with endomorphisms ring $D$; moreover we may assume $\operatorname{dim}_{D} V \geq 2$. Firstly we consider the case where $\operatorname{dim}_{D} V=t$ is finite. Thus $R$ contains some nontrivial idempotent element $e=e^{2}(e \neq 0,1)$. Of course, since $e(1-e)=0$ then $e$ is a zero-divisor, so $e \notin Z(R)$. By our main hypothesis, for all $r_{1}, \ldots, r_{n} \in R$ there exist $m=m\left(e, r_{1}, \ldots, r_{n}\right) \geq 1$ and $k=k\left(e, r_{1}, \ldots, r_{n}\right) \geq 1$ such that $\left[e^{m}, f\left(r_{1}, \ldots, r_{n}\right)\right]_{k}=0$, that is $\left[e, f\left(r_{1}, \ldots, r_{n}\right)\right]_{k}=0$. Hence, by the definition contained in Fact 1.7, $e \in H_{R}(f)$. Moreover, again by Fact 1.7, we have that either $f\left(x_{1}, \ldots, x_{n}\right)$ is central valued on $R$, or $H_{R}(f)=Z(R)$. In this last case we have the contradiction $e \in Z(R)$.

Assume now that $\operatorname{dim}_{D} V=\infty$. In [4] it is proved that the range of the polynomial $f\left(x_{1}, \ldots, x_{n}\right)$ is dense in $\operatorname{Hom}_{D}(V, V)$. So, given $D$-independent elements $u, v \in$ $V$, there exist $x, r_{1}, \ldots, r_{n} \in R$ such that $u x=u, v x=0, u f\left(r_{1}, \ldots, r_{n}\right)=v$ and $v f\left(r_{1}, \ldots, r_{n}\right)=v$. Then, for $k \geq 1$,

$$
0=u\left[x^{m}, f\left(r_{1}, \ldots, r_{n}\right)\right]_{k}=u f\left(r_{1}, \ldots, r_{n}\right)^{k}=v,
$$

which is a contradiction.

Lemma 2.4. Let $R$ be semiprime. If $R$ satisfies some polynomial identity, then $f\left(x_{1}, \ldots, x_{n}\right)$ is central valued on $R$.

Proof. Suppose first that $R$ is prime. Since $R$ is a $P I$-ring, then $Z(R) \neq\{0\}$ and the ring of central quotients of $R$, denoted by $Q=R Z^{-1}=\left\{r \alpha^{-1}: r \in R, \alpha \in Z(R)-\right.$ $\{0\}\}$, is a central simple algebra finite dimensional over its center. Thus $Q$ is primitive and satisfies the following condition: for all $x \in Q$ and for all $r_{1}, \ldots, r_{n} \in Q$ there exist integers $m=m\left(x, r_{1}, \ldots, r_{n}\right) \geq 1$ and $k=k\left(x, r_{1}, \ldots, r_{n}\right) \geq 1$ such that $\left[x^{m}, f\left(r_{1}, \ldots, r_{n}\right)\right]_{k}=0$. Hence by Lemma 2.3, $Q$ satisfies the polynomial identity $\left[f\left(x_{1}, \ldots, x_{n}\right), x_{n+1}\right]$, as well as $R$.

If $R$ is a semiprime ring, then $R$ is a subdirect sum of prime rings $R_{i}$. By the previous argument each $R_{i}$ satisfies $\left[f\left(x_{1}, \ldots, x_{n}\right), x_{n+1}\right]$, which implies that $R$ satisfies $\left[f\left(x_{1}, \ldots, x_{n}\right), x_{n+1}\right]$.

Lemma 2.5. If $R$ is semisimple then $f\left(x_{1}, \ldots, x_{n}\right)$ is central valued on $R$.

Proof. Since the Jacobson's radical $J(R)$ is zero, then $R$ is a subdirect product of primitive rings $R_{\gamma}=R / P_{\gamma}$, where any $P_{\gamma}$ is a prime ideal of $R$. By Lemmas 2.2 and 2.3 , it follows that $f\left(x_{1}, \ldots, x_{n}\right)$ is central valued in every $R_{\gamma}$. Therefore for all $r_{1}, \ldots, r_{n+1} \in R$ we have that $\left[f\left(r_{1}, \ldots, r_{n}\right), r_{n+1}\right] \in P_{\gamma}$, for any $\gamma$. Thus $\left[f\left(r_{1}, \ldots, r_{n}\right), r_{n+1}\right] \in \cap P_{\gamma}=(0)$, that is $R$ satisfies $\left[f\left(x_{1}, \ldots, x_{n}\right), x_{n+1}\right]$ and $f\left(x_{1}, \ldots, x_{n}\right)$ is central valued on $R$.

REMARK 2.6. In all that follows we may assume $J(R) \neq(0)$. 
Lemma 2.7. Let $P$ be a prime ideal of $R$ and assume that there exists $0 \neq a \in R$, such that $a^{2}=0$ and such that $a \notin P$. Then $R / P$ has no nonzero nil right ideal.

PRoof. Let $r_{1}, \ldots, r_{n} \in R$. Then there exist $m_{1}=m_{1}\left(a, r_{1}, \ldots, r_{n}\right) \geq 1, \quad k_{1}=$ $k_{1}\left(a, r_{1}, \ldots, r_{n}\right) \geq 1, m_{2}=m_{2}\left(a, r_{1}, \ldots, r_{n}\right) \geq 1$ and $k_{2}=k_{2}\left(a, r_{1}, \ldots, r_{n}\right) \geq 1$ such that both

$$
0=\left[f\left(r_{1} a, \ldots, r_{n} a\right)^{m_{1}}, f\left(a r_{1}, \ldots, a r_{n}\right)\right]_{k_{1}}=f\left(a r_{1}, \ldots, a r_{n}\right)^{k_{1}} \cdot f\left(r_{1} a, \ldots, r_{n} a\right)^{m_{1}}
$$

and

$$
\begin{aligned}
0 & =\left[\left(a f\left(r_{1} a, \ldots, r_{n} a\right)+f\left(r_{1} a, \ldots, r_{n} a\right)\right)^{m_{2}}, f\left(a r_{1}, \ldots, a r_{n}\right)\right]_{k_{2}} \\
& =f\left(a r_{1}, \ldots, a r_{n}\right)^{k_{2}} \cdot\left(a f\left(r_{1} a, \ldots, r_{n} a\right)^{m_{2}}+f\left(r_{1} a, \ldots, r_{n} a\right)^{m_{2}}\right) .
\end{aligned}
$$

In particular for $k=\max \left\{k_{1}, k_{2}\right\}$ and $m=\max \left\{m_{1}, m_{2}\right\}$, and from (2.1) and (2.2)

$$
\begin{aligned}
0 & =f\left(a r_{1}, \ldots, a r_{n}\right)^{k} \cdot\left(a f\left(r_{1} a, \ldots, r_{n} a\right)^{m}+f\left(r_{1} a, \ldots, r_{n} a\right)^{m}\right) \\
& =f\left(a r_{1}, \ldots, a r_{n}\right)^{k} \cdot a f\left(r_{1} a, \ldots, r_{n} a\right)^{m}=f\left(a r_{1}, \ldots, a r_{n}\right)^{k+m} a
\end{aligned}
$$

, and therefore $f\left(a r_{1}, \ldots, a r_{n}\right)^{k+m+1}=0$. By Fact 1.6 , the right ideal $\varrho=a R$ satisfies the identity $f\left(x_{1}, \ldots, x_{n}\right) x_{n+1}$. Since $a \notin P$ then $\varrho^{\prime}=\varrho / P$ is also a nonzero right ideal of $R / P$ which satisfies a polynomial identity. Suppose that $R / P$ has a nonzero nil right ideal $N$. Since $R / P$ is a prime ring, then there exists $b \in \varrho^{\prime}$ such that $b N$ is a nonzero nil right ideal. Moreover $b N \subseteq \varrho^{\prime}$ satisfies a polynomial identity, and this is a contradiction in a prime ring. Therefore $R / P$ has no nonzero nil right ideal, for all $P \in A$.

2.1. A reduced result. Here we prove a result which will be useful in the sequel. Firstly we state the following one, which is contained in [7, Lemma 1].

LEMMa 2.8. Let $R$ be a prime ring and let $\varrho$ be a nonzero right ideal of $R$ such that the left annihilator $l(\varrho)=\{x \in R: x \varrho=(0)\}$ is zero. If $\varrho$ satisfies a polynomial identity then $R$ also satisfies some polynomial identity.

Lemma 2.9. Let $R$ be a prime ring and suppose that for any $r_{1}, \ldots, r_{n} \in R$ there exists $m=m\left(r_{1}, \ldots, r_{n}\right) \geq 1$ such that $f\left(r_{1}, \ldots, r_{n}\right)^{m}$ is either zero or regular. If $R$ is not a domain, then $R$ satisfies some polynomial identity.

Proof. Firstly we note that if for any $r_{1}, \ldots, r_{n} \in R$ there exists $m=m\left(r_{1}, \ldots, r_{n}\right) \geq 1$ such that $f\left(r_{1}, \ldots, r_{n}\right)^{m}=0$, then by Fact $1.6, f\left(x_{1}, \ldots, x_{n}\right)$ is a polynomial identity for $R$ and the lemmas are proved. Assume that $R$ is not a domain. Hence there

exists $0 \neq a \in R$ such that $a^{2}=0$. Let $\varrho=a R$ and notice that the left annihilator $l(\varrho)$ is not zero. Thus $\varrho$ does not contain any regular element and so for any $r_{1}, \ldots, r_{n} \in \varrho$, there exists $m=m\left(r_{1}, \ldots, r_{n}\right) \geq 1$ such that $f\left(r_{1}, \ldots, r_{n}\right)^{m}=0$. In particular this also holds in $R_{1}=\varrho / l(\varrho) \cap \varrho$, which is a prime ring with no nonzero nil right ideal. Again by Fact 1.6, $f\left(x_{1}, \ldots, x_{n}\right)$ is a polynomial identity for $R_{1}$, that is $f\left(s_{1}, \ldots, s_{n}\right) \subseteq l(\varrho)$, for all $s_{1}, \ldots, s_{n} \in \varrho$. Therefore $\varrho$ satisfies the polynomial identity $f\left(x_{1}, \ldots, x_{n}\right) x_{n+1}$.

By Zorn's lemma there exists a nonzero right ideal $M$ of $R$ which is maximal with respect to the property that it satisfies $f\left(x_{1}, \ldots, x_{n}\right) x_{n+1}$. 
Now let $r \in R$ and $t_{1}, \ldots, t_{n+1} \in M$. Since $t_{i} r \in M$ (for all $i$ ), we have that $f\left(r t_{1}, \ldots, r t_{n}\right) r t_{n+1}=r f\left(t_{1} r, \ldots, t_{n} r\right) t_{n+1}=0$. This means that the right ideal $r M$ satisfies $f\left(x_{1}, \ldots, x_{n}\right) x_{n+1}$. Hence by [9, Theorem 6], $M+r M$ also satisfies some polynomial identity.

If $l(M+r M)=(0)$, then Lemma 2.9 is proved.

Suppose now that $l(M+r M) \neq(0)$. Then by the previous argument we have that $M+r M$ satisfies $f\left(x_{1}, \ldots, x_{n}\right) x_{n+1}$. Moreover by the maximality of $M$, it follows that $M+r M \subseteq M$, that is $r M \subseteq M$. This holds for all $r \in R$, implying that $M$ is a twosided ideal of $R$, which satisfies a polynomial identity. Therefore $R$ also satisfies a polynomial identity.

2.2. Proof of main theorem. In light of previous lemmas, we can now continue with the proof of our main results.

Proposition 2.10. If $R$ is a prime ring (without nil one-sided ideals), then $f\left(x_{1}, \ldots, x_{n}\right)$ is central valued on $R$.

Proof. By Lemmas 2.2 and 2.5 we may consider the case where $R$ is not a domain and $J(R) \neq(0)$. In addition, since $R$ and $J(R)$ satisfy the same polynomial identities, in order to prove that $f\left(x_{1}, \ldots, x_{n}\right)$ is central valued on $R$, we may replace $R$ by $J(R)$ (without loss of generality we consider $R=J(R))$. If $f\left(r_{1}, \ldots, r_{n}\right)$ is nilpotent for all $r_{1}, \ldots, r_{n} \in R$, then Fact 1.6 shows that $f\left(x_{1}, \ldots, x_{n}\right)$ is a polynomial identity for $R$. Hence we may suppose that there exist $r_{1}, \ldots, r_{n} \in R$ such that $c=f\left(r_{1}, \ldots, r_{n}\right)$ is not nilpotent, in other words $c^{m} \neq 0$ for all $m \geq 1$. Here we denote by $f(R)$ the set of all the evaluations of $f\left(x_{1}, \ldots, x_{n}\right)$ on $R$, that is $f(R)=\left\{f\left(r_{1}, \ldots, r_{n}\right): r_{i} \in R\right\}$.

We divide the proof into two cases.

Firstly we suppose that there exists an ideal $H$ of $R$ such that, for any $n \geq 1, c^{n} \notin H$. By Zorn's lemma there is an ideal $P_{c}$ of $R$ which is maximal with respect to the exclusion of all powers of $c$. In particular the ideal $P_{c}$ is a prime ideal of $R$ and, for any ideal $I$ of $R$ such that $P_{c} \varsubsetneqq I \subseteq R$, there exists $n=n(c) \geq 1$ such that $c^{n} \in I$.

Let $F=\left\{P_{c}: c \in f(R)\right.$ is not nilpotent $\}$ and consider the following partition of $F$ :

$$
\begin{gathered}
C=\left\{P_{c} \in F: \exists 0 \neq x \in R \text { such that } x^{2}=0, x \notin P_{c}\right\} \\
D=\left\{P_{c} \in F: \forall x \in R \text { such that } x^{2}=0, \text { then } x \in P_{c}\right\} .
\end{gathered}
$$

Let $M=\cap P_{c}$ for all $P_{c} \in F, \bar{C}=\cap P_{c}$ for all $P_{c} \in C$ and $\bar{D}=\cap P_{c}$ for all $P_{c} \in D$.

Note that if $r_{1}, \ldots, r_{n} \in M$ then $f\left(r_{1}, \ldots, r_{n}\right)$ is nilpotent (since if not, then $c=$ $f\left(r_{1}, \ldots, r_{n}\right) \notin P_{c}$, whereas $\left.c \in M \subseteq P_{c}\right)$. Moreover if $M \neq(0)$, then $f\left(x_{1}, \ldots, x_{n}\right)$ is nilpotent in the nonzero ideal $M$ of the prime ring $R$. Therefore, again from Fact 1.6, $f\left(x_{1}, \ldots, x_{n}\right)$ is central valued on $M$, as well as in $R$.

On the other hand if $M=(0)$, then $\bar{C} \cap \bar{D}=(0)$. Therefore $\bar{D}$ contains all the squarezero elements of $R$, and $\bar{C}$ contains no nonzero square-zero element. Since a ring with no nonzero square-zero element is a subdirect sum of a domain, then the ideal $\bar{C}$ is a subdirect sum of domains and by Lemma 2.2 we have that $f\left(x_{1}, \ldots, x_{n}\right)$ is central valued in $\bar{C}$, and so also in $R$. We may hence assume $\bar{C}=0$. 
In the last case, via the subdirect sum of $R / P_{c}$ for $P_{c} \in F$, we suppose that for any ideal $H \neq 0$ of $R$, there exists $m=m(H) \geq 1$ such that $c^{m} \in H$. Let $b \in f(R)$ and suppose that $b$ is neither nilpotent nor regular. We also define

$$
\begin{aligned}
& L_{b}=\left\{x \in R: x b^{n}=0, n=n(x) \geq 1\right\} \\
& T_{b}=\left\{x \in R: b^{n} x=0, n=n(x) \geq 1\right\} .
\end{aligned}
$$

Let $a \in L_{b}$. Since $b \in f(R)$, by the assumption of Theorem 1.1, there exist suitable $m=m(a, b) \geq 1$ and $k=k(a, b) \geq 1$ such that

$$
\left[b^{m},(1+a) b(1+a)^{-1}\right]_{k}=0 \quad \text { with } a b^{n}=0 \text { for some } n \geq 1,
$$

and in light of Fact 1.3 it also holds that

$$
\left[b^{m},(1+a) b^{n}(1+a)^{-1}\right]_{k}=0 \quad \text { with } a b^{n}=0 .
$$

Therefore

$$
\sum_{h=0}^{k}\left(\begin{array}{l}
k \\
h
\end{array}\right)(-1)^{h}\left((1+a) b^{n h}(1+a)^{-1}\right) b^{m}\left((1+a) b^{n(k-h)}(1+a)^{-1}\right)=0
$$

in other words

$$
\sum_{h=0}^{k-1}\left(\begin{array}{l}
k \\
h
\end{array}\right)(-1)^{h}\left((1+a) b^{n h}(1+a)^{-1}\right) b^{m}\left((1+a) b^{n(k-h)}(1+a)^{-1}\right)=b^{m+n k}
$$

and easy computations show that $b^{m+n k}(1+a)^{-1}=b^{m+n k}$, that is $b^{m+n k}=b^{m+n k}(1+a)$, that is $b^{m+n k} a=0$, that is $a \in T_{b}$. Analogously we can prove that $T_{b} \subseteq L_{b}$. Thus $T_{b}=L_{b}=I$ is a two-sided ideal of $R$. Since there exists a suitable $m \geq 1$ such that $c^{m} \in I$, it follows that $c$ is neither nilpotent nor regular. So by the above argument, there exists $m_{1} \geq 1$ such that $c^{m_{1}} \in L_{c}$, therefore there exists $m_{2} \geq 1$ such that $c^{m_{1}} c^{m_{2}}=0$, a contradiction.

Hence any element $b \in f(R)$ is either nilpotent or regular. Since we are considering the case when $R$ is not a domain, by Lemma 2.9, $R$ satisfies a polynomial identity and by Lemma $2.4 f\left(x_{1}, \ldots, x_{n}\right)$ is central valued on $R$.

The Proof of Theorem 1.1. By Lemma 2.1, we may consider only the case $\operatorname{char}(R)=$ 0 . Let

$$
\begin{gathered}
A=\left\{P \mid \text { there exists } 0 \neq x \in R \text { such that } x^{2}=0, x \notin P\right\} \\
B=\left\{P \mid \text { for any } x \in R \text { such that } x^{2}=0 \text { then } x \in P\right\}
\end{gathered}
$$

and $\bar{A}=\cap_{A} P, \bar{B}=\cap_{B} P$. Consider the diagonal map $\varphi: R \rightarrow \prod_{P \in A} R / P$. Since by Proposition $2.10 f\left(x_{1}, \ldots, x_{n}\right)$ is central valued on $R / P$, for all $P \in A$, 
then $f\left(x_{1}, \ldots, x_{n}\right)$ is central valued on $R / \operatorname{Ker}(\varphi)$, where $\operatorname{Ker}(\varphi)=\bigcap_{A} P=\bar{A}$ and $\bar{A} \cap \bar{B}=(0)$, by the semi-primeness of $R$. Therefore $\bar{B}$ contains all the square-zero elements of $R$, and $\bar{A}$ contains no nonzero square-zero element. In particular $\bar{A}$ is a subdirect sum of domains, so by Lemma $2.2 f\left(x_{1}, \ldots, x_{n}\right)$ is central valued on $\bar{A}$. Since $R / \bar{A}$ and $\bar{A}$ satisfy some polynomial identities, so does $R$ and we obtain the required conclusion by Lemma 2.4 .

\section{Acknowledgements}

The authors would like to thank the referee for helpful suggestions and careful reading of the paper. Moreover the authors would like to express their deepest gratitude to Professor Luisa Carini for her guidance throughout the revision of the paper.

\section{References}

[1] J. Bergen, 'Multilinear polynomials with power commuting values', Houston J. Math. 11(3) (1985), 283-292.

[2] M. Chacron, J. Lawrence and D. Madison, 'A note on radical extensions of rings', Canad. Math. Bull. 18(3) (1975), 423-425.

[3] C. L. Chuang and T. K. Lee, 'Rings with annihilator conditions on multilinear polynomials', Chinese J. Math. 24(2) (1996), 177-185.

[4] C. L. Chuang and T. K. Lee, 'Density of polynomial maps', Canad. Math. Bull. 53(2) (2010), 223-229.

[5] C. L. Chuang and J. S. Lin, 'On a conjecture by Herstein', J. Algebra 126 (1989), 119-138.

[6] V. De Filippis and O. M. Di Vincenzo, 'An Engel condition with derivation for multilinear polynomials in prime rings', Algebra Coll. 9(4) (2002), 361-374.

[7] O. M. Di Vincenzo and A. Valenti, 'On $n$th commutators with nilpotent or regular values in rings', Rend. Circ. Mat. Palermo SERIE II TOMO XL (1991), 453-464.

[8] I. N. Herstein, C. Procesi and M. Schacher, 'Algebraic valued functions on noncommutative rings', J. Algebra 36 (1975), 128-150.

[9] L. M. Rowen, 'General polynomial identities II', J. Algebra 38 (1976), 380-392.

VINCENZO DE FILIPPIS, DiSIA,

Department of Mathematics and Computer Science,

University of Messina, Viale F. Stagno D’Alcontres 31,

98166 Messina, Italy

e-mail: defilippis@unime.it

GIOVANNI SCUDO, Department of Mathematics and Computer Science, University of Messina, Viale F. Stagno D’Alcontres 31, 98166 Messina, Italy

e-mail: gscudo@unime.it 